\title{
ON SPACES OF MATRICES WITH A BOUNDED NUMBER OF EIGENVALUES
}

\author{
RAPHAEL LOEWY ${ }^{\dagger}$ AND NIZAR RADWAN ${ }^{\ddagger}$
}

\section{Dedicated to Hans Schneider on the occasion of his seventieth birthday}

\begin{abstract}
The following problem, originally proposed by Omladič and Šemrl [Linear Algebra Appl., 249:29-46 (1996)], is considered. Let $k$ and $n$ be positive integers such that $k<n$. Let $L$ be a subspace of $M_{n}(F)$, the space of $n \times n$ matrices over a field $F$, such that each $A \in L$ has at most $k$ distinct eigenvalues (in the algebraic closure of $F$ ). Then, what is the maximal dimension of $L$. Omladič and Šemrl assumed that $F=\mathbb{C}$ and solved the problem for $k=1, k=2$ and $n$ odd, and $k=n-1$ (under a mild assumption). In this paper, their results for $k=1$ and $k=n-1$ are extended to any $F$ such that $\operatorname{char}(F)=0$, and a solution for $k=2$ and any $n$, and for $k=3$ is given.
\end{abstract}

Key words. Distinct eigenvalues; cornal ordering; irreducible polynomial.

AMS subject classifications. 15A42; $15 \mathrm{~A} 45$.

1. Introduction. Let $\mathrm{F}$ be a field. Let $M_{m, n}(F)$ denote the space of all $m \times n$ matrices over $F$ Tand $S_{n}(F)$ denote the space of all $n \times n$ symmetric matrices over $F$. Let $M_{n}(F)=M_{n, n}(F)$. In recent years there have been many works considering spaces of matrices which satisfy certain properties. For example $\mathrm{C}$ given a positive integer $k \Gamma$ what can be said about a subspace $L$ of $M_{m, n}(F)$ (or $S_{n}(F)$ ) if every nonzero matrix in $L$ has rank $k$. Or $\Gamma$ what can be said if every matrix in $L$ has rank bounded above by $k$. One can consider also spectral properties. Gerstenhaber [G] showed that every subspace $L$ of $M_{n}(F)$ consisting of nilpotent matrices has dimension bounded by $\left(\begin{array}{c}n \\ 2\end{array}\right)$ and if $|F| \geq n$ and $\operatorname{dim} L=\left(\begin{array}{c}n \\ 2\end{array}\right) \Gamma$ then $L$ is conjugate to the algebra of strictly upper triangular matrices; see also [Se] and [BC].

In this paper we consider the following problem. Let $k$ and $n$ be positive integers such that $k<n$. Let $L$ be a subspace of $M_{n}(F)$ such that every $A \in L$ has at most $k$ distinct eigenvalues (in the algebraic closure of $F$ ). Then $\Gamma$ what is the maximal dimension of $L$ ? This problem was proposed by Omladič and Šemrl [OS]. Atkinson [A] considered a subspace $L$ of $M_{n}(F)$ with the property that every $A \in L$ has zero eigenvalue of (algebraic) multiplicity at least $r$ Twhere $1 \leq r<n$; see Theorem 2.7 . Clearly such $L$ has the property that every $A \in L$ has at most $n-r+1$ distinct eigenvalues. Omladič and Šemrl [OS] obtained the following results.

THEOREM 1.1. (a) Let $L$ be a subspace of $M_{n}(\mathbb{C})$ such that every $A \in L$ has only one eigenvalue. Then, $\operatorname{dim} L \leq\left(\begin{array}{c}n \\ 2\end{array}\right)+1$.

(b) Let $L$ be a subspace of $M_{n}(\mathbb{C})$ where $n$ is odd and such that every $A \in L$ has at most 2 distinct eigenvalues. Then, $\operatorname{dim} L \leq\left(\begin{array}{l}n \\ 2\end{array}\right)+2$.

(c) Let $L$ be a subspace of $M_{n}(\mathbb{C})$ such that every $A \in L$ has at most $n-1$ distinct

${ }^{*}$ Received by the editors 7 November 1997. Final manuscript accepted on 31 August 1998. Handling editor: Volker Mehrmann.

${ }^{\dagger}$ The research of this author was supported by Technion V.P.R. Fund-Japan Technion Society Research Fund.

$\ddagger$ Department of Mathematics, Technion - Israel Institute of Technology, Haifa 32000, Israel (loewy@techunix.technion.ac.il, rnizar@techunix.technion.ac.il). 
eigenvalues. Assume that there exists some $A \in L$ which has exactly $n-1$ distinct eigenvalues. Then,

$$
\operatorname{dim} L \leq\left(\begin{array}{l}
n \\
2
\end{array}\right)+\left(\begin{array}{c}
n-1 \\
2
\end{array}\right)+1
$$

In each case Omladič and Šemrl also determined the structure of every $L$ for which the maximum dimension is attained. Of course $\Gamma$ (a) is closely related to the nilpotent case. It is our purpose to extend the results of Theorem 1.1 to any field such that $\operatorname{char}(F)=0$ Tcomplete the case $k=2$ for any $n$ Tand give a solution to the case $k=3$. The results obtained seem to suggest the following conjecture.

CONJECTURE 1.2. Let $k$ and $n$ be integers, $k<n$, and let $F$ be a field such that $\operatorname{char}(F)=0$. Let $L$ be a subspace of $M_{n}(F)$ such that every $A \in L$ has at most $k$ distinct eigenvalues. Then,

$$
\operatorname{dim} L \leq\left(\begin{array}{l}
n \\
2
\end{array}\right)+\left(\begin{array}{l}
k \\
2
\end{array}\right)+1 .
$$

In Section 2 we bring some preliminary notations and results $\Gamma$ while in Section 3 we give our main results of this paper.

We assume throughout (unless explicitly stated otherwise) that $F$ is a field such that char $(F)=0$ and consider $\alpha=\left(\alpha_{1}, \ldots, \alpha_{m}\right)$ as an arbitrary point in $F^{m}$ or as a vector of indeterminates $\Gamma$ according to our convenience.

2. Preliminary Notations and Results. Let $A \in M_{n}(F)$. We denote by $\sigma(A)$ the set of all eigenvalues of $A$ in the algebraic closure of $F$. We denote by $\#(\sigma(A))$ the number of distinct eigenvalues of $A$. A subspace $V$ of $M_{n}(F)$ is said to be a $\bar{k}$-spect subspace provided that $k=\max \{\#(\sigma(A)): A \in V\}$. Let $\left\{A_{1}, A_{2}, \ldots, A_{m}\right\}$ be a set of $m$ linearly independent matrices in $M_{n}(F)$ and let $p(t, \alpha)=\operatorname{det}\left(t I-\sum_{i=1}^{m} \alpha_{i} A_{i}\right)$. We consider $p(t, \alpha)$ as a polynomial in $t$ with coefficients in $F\left[\alpha_{1}, \ldots, \alpha_{m}\right] \Gamma$ that is $\Gamma$ a polynomial in $F\left[\alpha_{1}, \ldots, \alpha_{m}\right][t]$ which is a unique factorization domain. Then $p(t, \alpha)$ can be split in $F\left[\alpha_{1}, \ldots, \alpha_{m}\right][t]$ as follows:

$$
p(t, \alpha)=p_{1}^{k_{1}}(t, \alpha) \cdots p_{\ell}^{k_{\ell}}(t, \alpha),
$$

where $p_{j}(t, \alpha)$ are monic distinct irreducible polynomials.

Denote $n_{j}=\operatorname{deg}\left(p_{j}\right)$. Then $p_{j}(t, \alpha)$ is of the form

$$
p_{j}(t, \alpha)=t^{n_{j}}+q_{j 1} t^{n_{j}-1}+\cdots+q_{j, n_{j}-1} t+q_{j, n_{j}},
$$

where $q_{j, r}=q_{j, r}\left(\alpha_{1}, \ldots, \alpha_{m}\right)$ is a homogeneous polynomial in $\alpha_{1}, \ldots, \alpha_{m}$ of degree $r$ $\left(r=1,2, \ldots, n_{j}\right)$.

$$
\text { Clearly } \sum_{j=1}^{\ell} k_{j} n_{j}=n \text {. }
$$


Denote $k=\sum_{j=1}^{\ell} n_{j}$. If $k<n$ and $V$ is spanned by $\left\{A_{1}, \ldots, A_{m}\right\} \Gamma$ then for every $A \in V \Gamma \#(\sigma(A)) \leq k$. In the following lemma we show that there exists $A \in V$ such that $\#(\sigma(A))=k$ That is $V$ is a $\bar{k}$-spect subspace of $M_{n}(F)$.

Lemma 2.1. Let $F$ be a field with $\operatorname{char}(F)=0$. Let $V$ be a subspace of $M_{n}(F)$ and let $\left\{A_{1}, \ldots, A_{m}\right\}$ be a basis of $V$. Let

$$
p(t, \alpha)=\operatorname{det}\left(t I_{n}-\sum_{i=1}^{m} \alpha_{i} A_{i}\right)
$$

be split into monic distinct irreducible polynomials in $F\left[\alpha_{1}, \ldots, \alpha_{m}\right][t]$

$$
p(t, \alpha)=p_{1}^{k_{1}}(t, \alpha) \cdots p_{\ell}^{k_{\ell}}(t, \alpha) .
$$

Denote $n_{j}=\operatorname{deg} p_{j}(j=1,2, \ldots, \ell)$ and $k=\sum_{j=1}^{\ell} n_{j}$. Then there exists a nonzero polynomial $\varphi(\alpha)$ in $F\left[\alpha_{1}, \ldots, \alpha_{m}\right]$ such that any $\hat{\alpha} \in F^{m}$ for which $\varphi(\hat{\alpha}) \neq 0$ satisfies $\#\left(\sigma\left(\sum_{i=1}^{m} \hat{\alpha}_{i} A_{i}\right)\right)=k$.

Proof. We can assume $k_{j}=1(j=1,2, \ldots, \ell)$. We shall proceed by induction with respect to $\ell$. If $\ell=1 \Gamma$ then $p(t, \alpha)=p_{1}(t, \alpha)$ is irreducible. Thus $p_{1}(t, \alpha)$ and its derivative $p_{1}^{\prime}(t, \alpha)$ with respect to $t$ are relatively prime. Hence $\Gamma$ there exist polynomials $q_{1}(t, \alpha)$ and $q_{2}(t, \alpha)$ in $F\left[\alpha_{1}, \ldots, \alpha_{m}\right][t]$ and a nonzero polynomial $\varphi(\alpha) \in$ $F\left[\alpha_{1}, \ldots, \alpha_{m}\right]$ such that

$$
q_{1}(t, \alpha) p_{1}(t, \alpha)+q_{2}(t, \alpha) p_{1}^{\prime}(t, \alpha)=\varphi(\alpha) .
$$

Now $\Gamma$ for any $\hat{\alpha} \in F^{m}$ for which $\varphi(\hat{\alpha}) \neq 0 \Gamma p_{1}(t, \hat{\alpha})$ and $p_{1}^{\prime}(t, \hat{\alpha})$ are relatively prime as polynomials in $F[t]$. Therefore $\Gamma p_{1}(t, \hat{\alpha})$ has $k=n_{1}$ distinct roots $\Gamma$ which implies $\#\left(\sigma\left(\sum_{i=1}^{m} \hat{\alpha}_{i} A_{i}\right)\right)=k$.

Assume $\ell>1$. As we have seen $\Gamma$ there exists a nonzero polynomial $\varphi_{1}(\alpha) \in$ $F\left[\alpha_{1}, \ldots, \alpha_{m}\right]$ such that any $\hat{\alpha} \in F^{m}$ for which $\varphi_{1}(\hat{\alpha}) \neq 0$ implies that $p_{1}(t, \hat{\alpha})$ has $n_{1}$ distinct roots. By the induction hypothesis there exists a nonzero polynomial $\varphi_{2}(\alpha) \in F\left[\alpha_{1}, \ldots, \alpha_{m}\right]$ such that for any $\hat{\alpha} \in F^{m}$ for which $\varphi_{2}(\hat{\alpha}) \neq 0$ the polynomial $p_{2}(t, \alpha) \cdots p_{\ell}(t, \alpha)$ has $\sum_{j=2}^{\ell} n_{j}$ distinct roots.

Since $p_{1}(t, \alpha)$ and $p_{2}(t, \alpha) \cdots p_{\ell}(t, \alpha)$ are relatively prime $\Gamma$ there exist $\hat{q}_{1}(t, \alpha)$ and $\hat{q}_{2}(t, \alpha)$ in $F\left[\alpha_{1}, \ldots, \alpha_{m}\right][t]$ such that

$$
\hat{q}_{1}(t, \alpha) p_{1}(t, \alpha)+\hat{q}_{2}(t, \alpha) p_{2}(t, \alpha) \cdots p_{\ell}(t, \alpha)=\varphi_{3}(\alpha),
$$

where $\varphi_{3}(\alpha)$ is a nonzero polynomial in $F\left[\alpha_{1}, \ldots, \alpha_{m}\right]$. 
For any $\hat{\alpha} \in F^{m}$ for which $\varphi_{3}(\hat{\alpha}) \neq 0 \Gamma p_{1}(t, \hat{\alpha})$ and $p_{2}(t, \hat{\alpha}) \cdots p_{\ell}(t, \hat{\alpha})$ are relatively prime as polynomials in $F[t]$. Hence $\Gamma$ they have no common root.

Define $\varphi(\alpha)=\varphi_{1}(\alpha) \varphi_{2}(\alpha) \varphi_{3}(\alpha)$. For any $\hat{\alpha} \in F^{m}$ for which $\varphi(\hat{\alpha}) \neq 0$ Tthe polynomial $p(t, \hat{\alpha})$ has $k$ distinct roots. Thus $\Gamma A=\sum_{i=1}^{m} \hat{\alpha}_{i} A_{i}$ satisfies $\#(\sigma(A))=k$.

The next lemma allows us to obtainTunder some conditionTan $(m-1)$-dimensional $\bar{\ell}$-spect subspace from an $m$-dimensional $\bar{k}$-spect subspace where $\ell \leq k-1$.

LEMMA 2.2. Let $F$ be a field with $\operatorname{char}(F)=0$. Let $V$ be a $\bar{k}$-spect subspace of $M_{n}(F)$ and let $\left\{A_{1}, \ldots, A_{m}\right\}$ be a basis of $V$. Suppose that in the splitting of

$$
p(t, \alpha)=\operatorname{det}\left(t I_{n}-\sum_{i=1}^{m} \alpha_{i} A_{i}\right)
$$

into monic irreducible polynomials, there occur two distinct linear polynomials. Then, $V$ contains an $\bar{\ell}$-spect subspace of dimension $m-1$, where $\ell \leq k-1$.

Proof. Let

$$
p(t, \alpha)=p_{1}^{k_{1}}(t, \alpha) p_{2}^{k_{2}}(t, \alpha) \cdots p_{r}^{k_{r}}(t, \alpha),
$$

where $p_{j}(t, \alpha)$ are distinct monic irreducible polynomials in $F\left[\alpha_{1}, \ldots, \alpha_{m}\right][t]$. Suppose $p_{1}$ and $p_{2}$ are linear. We can write

$$
p_{1}(t, \alpha)=t-\mu_{1}(\alpha) \text { and } p_{2}(t, \alpha)=t-\mu_{2}(\alpha) \text {, }
$$

where

$$
\mu_{1}(\alpha)=\sum_{i=1}^{m} a_{i} \alpha_{i} \quad \text { and } \quad \mu_{2}(\alpha)=\sum_{i=1}^{m} b_{i} \alpha_{i}
$$

Clearly $a_{i}$ and $b_{i}$ are eigenvalues of $A_{i}(i=1, \ldots, m)$.

Since $p_{1}(t, \alpha)$ and $p_{2}(t, \alpha)$ are relatively prime $\Gamma$ there exists $i_{0} \Gamma 1 \leq i_{0} \leq m \Gamma$ such that $a_{i_{0}} \neq b_{i_{0}}$. For any $\tilde{\alpha}=\left(\alpha_{1}, \ldots, \alpha_{i_{0}-1}, \alpha_{i_{0}+1}, \ldots, \alpha_{m}\right) \in F^{m-1}$ there exists a unique $\hat{\alpha}_{i_{0}} \in F$ which satisfies

$$
\sum_{\substack{i=1 \\ i \neq i_{0}}}^{m} \alpha_{i}\left(a_{i}-b_{i}\right)+\hat{\alpha}_{i_{0}}\left(a_{i_{0}}-b_{i_{0}}\right)=0
$$

Denote $B_{i}=A_{i}-\frac{a_{i}-b_{i}}{a_{i_{0}}-b_{i_{0}}} A_{i_{0}} \Gamma i=1, \ldots, m \Gamma i \neq i_{0}$ and $\hat{V}=\operatorname{span}\left\{B_{i}\right\}_{\substack{i=1 \\ i \neq i_{0}}}^{m}$. Clearly $\Gamma$ $\hat{V}$ is a subspace of $V$ of dimension $m-1$. For $\tilde{\alpha}=\left(\alpha_{1}, \ldots, \alpha_{i_{0}-1}, \alpha_{i_{0}+1}, \ldots, \alpha_{m}\right)$ in $F^{m-1}$ we have

$$
\sum_{\substack{i=1 \\ i \neq i_{0}}}^{m} \alpha_{i} B_{i}=\sum_{\substack{i=1 \\ i \neq i_{0}}}^{m} \alpha_{i} A_{i}-\frac{1}{a_{i_{0}}-b_{i_{0}}} \sum_{\substack{i=1 \\ i \neq i_{0}}}^{m} \alpha_{i}\left(a_{i}-b_{i}\right) A_{i_{0}}=\sum_{\substack{i=1 \\ i \neq i_{0}}}^{m} \alpha_{i} A_{i}+\hat{\alpha}_{i_{0}} A_{i_{0}}
$$

Hence $\Gamma \mu_{1}(\alpha)=\mu_{2}(\alpha)$ for all $\alpha=\left(\alpha_{1}, \ldots, \alpha_{i_{0}-1}, \hat{\alpha}_{i_{0}}, \alpha_{i_{0}+1}, \ldots, \alpha_{m}\right) \in F^{m}$. 
It follows from Lemma 2.1 that $\hat{V}$ is an $\bar{\ell}$-spect subspace $\Gamma$ where $\ell \leq k-1$.

We introduce now a linear ordering on the elements of $[n] \times[n] \bar{\Gamma}$ where $[n]=$ $\{1,2, \ldots, n\}$.

Definition 2.3. A linear ordering $\Gamma$ on $[n] \times[n]$ is said to be a cornal ordering if it satisfies the following three conditions.

(I) $\left(i_{1}, 1\right)<\left(i_{2}, 1\right)$ iff $i_{1}>i_{2}$.

(II) $\left(1, j_{1}\right) \underset{\Gamma}{<}\left(1, j_{2}\right)$ iff $j_{1}<j_{2}$.

(III) $(p, 1) \underset{\Gamma}{<}(i, j) \underset{\Gamma}{<}(1, q)$ for all $i, j, p>1$ and $q \geq 1$

Definition 2.4. Let $\Gamma$ be a linear ordering on $[n] \times[n]$ and let $A=\left(a_{i j}\right)$ be a nonzero matrix in $M_{n}(F)$. We denote $d_{\Gamma}(A)=(p, q)$ Twhere $(p, q)=\min \left\{(i, j), a_{i j} \neq\right.$ $0\}$ and the minimum is taken with respect to $\Gamma$.

Let $V$ be a subspace of $M_{n}(F)$ and let $\left\{A_{1}, \ldots, A_{m}\right\}$ be a basis of $V$. Let $\Gamma$ be a linear ordering on $[n] \times[n]$. We may think of a matrix in $M_{n}(F)$ as an $n^{2}$-tuple taken in the order specified by $\Gamma$. Performing Gaussian elimination on $\left\{A_{1}, \ldots, A_{m}\right\}$ with respect to $\Gamma$ Twe get a basis $\left\{B_{1}, \ldots, B_{m}\right\}$ of $V$ which satisfies $d_{\Gamma}\left(B_{i}\right)<_{\Gamma} d_{\Gamma}\left(B_{j}\right)$ for all $1 \leq i<j \leq m$ and each matrix $B_{i}$ has an entry equal to 1 in the position $d_{\Gamma}\left(B_{i}\right)$. We call that 1 the leading 1 of $B_{i}$ with respect to $\Gamma$. We may assume that for all $i, j=1, \ldots, m \Gamma i \neq j \Gamma B_{j}$ has a zero entry in the position of the leading 1 of $B_{i}$.

DEFINITION 2.5. Let $V$ be a subspace of $M_{n}(F)$ and let $\Gamma$ be a linear ordering on $[n] \times[n]$. We say that the basis $\left\{B_{1}, \ldots, B_{m}\right\}$ of $V$ is a $\Gamma$-ordered basis if it is obtained from some basis of $V$ by Gaussian elimination with respect to the order $\Gamma$.

We use the technique of the leading one's described above to obtain the following useful lemma.

LEMMA 2.6. Let $\Gamma$ be a cornal ordering on $[n] \times[n]$ and let $A_{r}$ and $A_{s}$ be matrices in $M_{n}(F)$ such that $d_{\Gamma}\left(A_{r}\right)=(\ell, 1)$ and $d_{\Gamma}\left(A_{s}\right)=(1, \ell)$ for some $2 \leq \ell \leq n$. Then the coefficient of $t^{n-2}$ in

$$
p\left(t, \alpha_{r}, \alpha_{s}\right)=\operatorname{det}\left[t I_{n}-\left(\alpha_{r} A_{r}+\alpha_{s} A_{s}\right)\right]
$$

must depend on $\alpha_{s}$.

Proof. The coefficient of $t^{n-2}$ in $p\left(t, \alpha_{r}, \alpha_{s}\right)$ equals $\sigma_{2}\left(\alpha_{r} A_{r}+\alpha_{s} A_{s}\right) \Gamma$ where $\sigma_{2}\left(\alpha_{r} A_{r}+\alpha_{s} A_{s}\right)$ is the sum of the principal minors of order 2 of the matrix $\alpha_{r} A_{r}+$ $\alpha_{s} A_{s}$. It follows from the choice of $\Gamma$ that the term $-\alpha_{r} \cdot \alpha_{s}$ must appear in $\sigma_{2}\left(\alpha_{r} A_{r}+\right.$ $\left.\alpha_{s} A_{s}\right)$. प

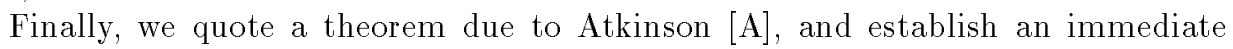
corollary.

Theorem 2.7. Let $F$ be a field, $|F| \geq n$, let $r$ be an integer, $r<n$, and let $V$ be a subspace of $M_{n}(F)$ with the property that every $A \in V$ has at least $r$ zero eigenvalues. Then $\operatorname{dim} V \leq \frac{1}{2} r(r-1)+n(n-r)$.

REMARK 2.8. We notice that if $A \in M_{n}(F)$ has at least $r$ zero eigenvaluesTthen $\#(\sigma(A)) \leq n-r+1$. From [A] we can deduce that if equality holds in Theorem 2.7Tthen there exists $A \in V$ such that $\#(\sigma(A))=n-r+1$. Therefore $V$ is $\bar{k}$-spect $\Gamma$ 
where $k=n-r+1$. For $k=n-r+1$ sstraightforward computation shows that

$$
\frac{1}{2} r(r-1)+n(n-r)=\left(\begin{array}{l}
n \\
2
\end{array}\right)+\left(\begin{array}{l}
k \\
2
\end{array}\right) .
$$

Thus $\Gamma$ if we adjoin the identity matrix to a subspace of the maximum dimension having that property $\Gamma$ we get a $\bar{k}$-spect subspace of the maximum dimension in our conjecture.

COROLLARY 2.9. Let $V$ be a subspace of $M_{n}(F), \operatorname{char}(F)=0$, that includes the identity matrix. Let $\left\{A_{1}, \ldots, A_{m}\right\}$ be a basis of $V$ in which $A_{m}=I_{n}$. Denote

$$
p(t, \alpha)=\operatorname{det}\left(t I_{n}-\sum_{i=1}^{m} \alpha_{i} A_{i}\right)
$$

Assume $p(t, \alpha)$ splits in $F\left[\alpha_{1}, \ldots, \alpha_{m}\right][t]$ in the form $p(t, \alpha)=Q(t, \alpha) \cdot q^{r}(t, \alpha)$, where $q(t, \alpha)$ is monic and linear. Then, $\operatorname{dim} V \leq \frac{1}{2} r(r-1)+n(n-r)+1$.

Proof. Write $q(t, \alpha)=t-\mu(\alpha)$ Twhere

$$
\mu(\alpha)=\sum_{i=1}^{m} a_{i} \alpha_{i}
$$

Since $a_{i}$ is an eigenvalue of $A_{i}$ Twe have $a_{m}=1$.

Define $B_{i}=A_{i}-a_{i} I_{n} \Gamma i=1,2, \ldots, m$ and $\hat{V}=\operatorname{span}\left\{B_{i}\right\}_{i=1}^{m}$. Clearly $\operatorname{dim} \hat{V}=$ $m-1$. For every $\alpha_{i} \in F \Gamma i=1,2, \ldots, m-1 \Gamma$

$$
\sum_{i=1}^{m-1} \alpha_{i} B_{i}=\sum_{i=1}^{m-1} \alpha_{i}\left(A_{i}-a_{i} I_{n}\right)=\sum_{i=1}^{m-1} \alpha_{i} A_{i}-\sum_{i=1}^{m-1} \alpha_{i} a_{i} I_{n} .
$$

Now $\Gamma$ denote $\bar{p}(t, \bar{\alpha})=\operatorname{det}\left(t I_{n}-\sum_{i=1}^{m-1} \alpha_{i} B_{i}\right) \Gamma$ where $\bar{\alpha}=\left(\alpha_{1}, \ldots, \alpha_{m-1}\right) \in F^{m-1}$. Then $\bar{p}(t, \bar{\alpha})$ splits in the form

$$
\bar{p}(t, \bar{\alpha})=\bar{Q}(t, \bar{\alpha}) \cdot t^{r} .
$$

Hence Tevery $A \in \hat{V}$ has at least $r$ zero eigenvalues. By Theorem 2.7

$$
\operatorname{dim} \hat{V} \leq \frac{1}{2} r(r-1)+n(n-r)
$$

thus $\Gamma \operatorname{dim} V \leq \frac{1}{2} r(r-1)+n(n-r)+1$.

3. Main Results. The upper bound of $\overline{1}$-spect subspaces is achieved by the following result.

THEOREM 3.1. Let $F$ be a field with $\operatorname{char}(F)=0$. Let $V$ be a $\overline{1}$-spect subspace of $M_{n}(F)$. Then $\operatorname{dim} V \leq \frac{n(n-1)}{2}+1$ and if equality holds then $V$ is conjugate to the space of all upper triangular matrices having equal diagonal entries. 
Proof. We may assume that $I_{n} \in V$. Let $\left\{A_{1}, \ldots, A_{m}\right\}$ be a basis of $V$. Denote

$$
p(t, \alpha)=\operatorname{det}\left(t I_{n}-\sum_{i=1}^{m} \alpha_{i} A_{i}\right)
$$

By Lemma $2.1 \Gamma p(t, \alpha)$ splits in $F\left[\alpha_{1}, \ldots, \alpha_{m}\right][t]$ in the following form:

$$
p(t, \alpha)=\left(t-\sum_{i=1}^{m} a_{i} \alpha_{i}\right)^{n}
$$

For $i=1, \ldots, m$ define

$$
B_{i}=A_{i}-a_{i} I_{n}
$$

Let $\hat{V}=\operatorname{span}\left\{B_{i}\right\}_{i=1}^{m}$. Clearly $\hat{V}$ is a space of nilpotent matrices of dimension $m-1$. The assertion follows from Gerstenhaber's result.

For $\overline{2}$-spect subspaces we have the following theorem.

THEOREM 3.2. Let $F$ be a field with $\operatorname{char}(F)=0$, and $n \geq 3$. Let $V$ be a $\overline{2}$-spect subspace of $M_{n}(F)$. Then

$$
\operatorname{dim} V \leq \frac{n(n-1)}{2}+2
$$

Proof. We may assume $I_{n} \in V$. Let $\Gamma$ be a cornal ordering on $[n] \times[n]$ and let $\left\{A_{1}, \ldots, A_{m}\right\}$ be a $\Gamma$-ordered basis of $V$. Denote

$$
p(t, \alpha)=\operatorname{det}\left(t I_{n}-\sum_{i=1}^{m} \alpha_{i} A_{i}\right) .
$$

By Lemma 2.1 $\Gamma p(t, \alpha)$ splits in $F\left[\alpha_{1}, \ldots, \alpha_{m}\right][t]$ in one of the following two forms. Case 1: $p(t, \alpha)=p_{1}^{k_{1}}(t, \alpha) \cdot p_{2}^{k_{2}}(t, \alpha) \Gamma$ where $p_{1}(t, \alpha)$ and $p_{2}(t, \alpha)$ are linear and distinct. Hence $\mathrm{C}$ by Lemma $2.2 \Gamma \mathrm{V}$ contains a $\overline{1}$-spect subspace of dimension $m-1$. Now $\Gamma$ the assertion follows from Theorem 3.1.

Case 2: $p(t, \alpha)=q^{n / 2}(t, \alpha)$ (in this case $\mathrm{n}$ must be even) $\Gamma$ where $q(t, \alpha)$ is quadratic and irreducible of the form

$$
q(t, \alpha)=t^{2}+q_{1}(\alpha) t+q_{2}(\alpha),
$$

where $q_{1}(\alpha)$ and $q_{2}(\alpha)$ are homogeneous in $\alpha_{1}, \ldots, \alpha_{m}$.

Claim: There do not exist $1 \leq r<s \leq m$ and $2 \leq \ell \leq n$ such that $A_{r}$ and $A_{s}$ have leading one's with respect to $\Gamma$ Tin the positions $(\ell, 1)$ and $(1, \ell)$ respectively.

Proof of claim: Suppose $d_{\Gamma}\left(A_{r}\right)=(\ell, 1)$ and $d_{\Gamma}\left(A_{s}\right)=(1, \ell)$ for some $2 \leq \ell \leq n$ and $1 \leq r<s \leq m$. Denote $\hat{\alpha}=\alpha_{r} e_{r}+\alpha_{s} e_{s} \in F^{m}$. Thus

$$
q(t, \hat{\alpha})=t^{2}+q_{1}\left(\alpha_{r}, \alpha_{s}\right) t+q_{2}\left(\alpha_{r}, \alpha_{s}\right),
$$


where $q_{1}\left(\alpha_{r}, \alpha_{s}\right)$ and $q_{2}\left(\alpha_{r}, \alpha_{s}\right)$ are of the form

$$
\begin{aligned}
& q_{1}\left(\alpha_{r}, \alpha_{s}\right)=a_{1} \alpha_{r}+b_{1} \alpha_{s} \\
& q_{2}\left(\alpha_{r}, \alpha_{s}\right)=a_{11} \alpha_{r}^{2}+a_{12} \alpha_{r} \alpha_{s}+a_{22} \alpha_{s}^{2} \\
& a_{1}, b_{1}, a_{11}, a_{12}, a_{22} \in F
\end{aligned}
$$

Since $q_{1}\left(0, \alpha_{s}\right)=q_{2}\left(0, \alpha_{s}\right)=0$ for all $\alpha_{s}$ Tthe coefficients $b_{1}$ and $a_{22}$ must vanish.

We have $\operatorname{det}\left(\alpha_{r} A_{r}+\alpha_{s} A_{s}\right)=\left[q_{2}\left(\alpha_{r}, \alpha_{s}\right)\right]^{n / 2}=\left(a_{11} \alpha_{r}^{2}+a_{12} \alpha_{r} \alpha_{s}\right)^{n / 2}$ Tbut on the other hand $\operatorname{det}\left(\alpha_{r} A_{r}+\alpha_{s} A_{s}\right)$ is either linear or independent of $\alpha_{s}$. Hence $\Gamma a_{12}$ must vanish also. It follows that $q_{1}\left(\alpha_{r}, \alpha_{s}\right)$ and $q_{2}\left(\alpha_{r}, \alpha_{s}\right)$ are independent of $\alpha_{s}$ which contradicts Lemma 2.6.

Now $T$ denote

$$
S=\left\{A_{i}: d_{\Gamma}\left(A_{i}\right)=(1, q) \text { or } d_{\Gamma}\left(A_{i}\right)=(p, 1) \text { for some } 2 \leq p, q \leq n\right\}
$$

By our claim $|S| \leq n-1$.

Define $V_{1}=\operatorname{span}\left(\left\{A_{1}, \ldots, A_{m}\right\} \backslash S\right)$. We have $\operatorname{dim} V_{1} \geq \operatorname{dim} V-(n-1)$.

Let $\hat{V}$ denote the subspace of $M_{n-1}(F)$ obtained from $V_{1}$ by deleting the first row and column of every matrix in $V_{1}$.

i) If there exists $1 \leq j \leq m$ such that $d_{\Gamma}\left(A_{j}\right)=(1,1)$ Tthen $\operatorname{dim} \hat{V}=\operatorname{dim} V_{1}-1$ and $\hat{V}$ is $\overline{1}$-spect subspace of $M_{n-1}(F)$. By Theorem 3.1

$$
\operatorname{dim} \hat{V} \leq \frac{(n-1)(n-2)}{2}+1
$$

Hence $\Gamma \operatorname{dim} V \leq \frac{(n-1)(n-2)}{2}+1+(n-1)+1=\frac{n(n-1)}{2}+2$.

ii) If there is no $1 \leq j \leq m$ such that $d_{\Gamma}\left(A_{j}\right)=(1,1) \Gamma$ then $\operatorname{dim} \hat{V}=\operatorname{dim} V_{1}$. Since $\hat{V}$ is either $\overline{2}$-spect or $\overline{1}$-spect subspace of $M_{n-1}(F)$ and $n-1$ is oddTit follows from the proof of Case 1 and Theorem 3.1 that

$$
\operatorname{dim} \hat{V} \leq \frac{(n-1)(n-2)}{2}+2
$$

which yields the assertion of the theorem. $\square$

For the case $k=3$ we have the following result.

Theorem 3.3. Let $F$ be a field with $\operatorname{char}(F)=0$ and $n \geq 4$. Let $V$ be a $\overline{3}$-spect subspace of $M_{n}(F)$. Then $\operatorname{dim} V \leq \frac{n(n-1)}{2}+4$.

Proof. As in our proof of Theorem 3.2Twe may assume that $I_{n} \in V$ and $\left\{A_{1}, \ldots, A_{m}\right\}$ is a $\Gamma$-ordered basis of $V$ Twhere $\Gamma$ is a cornal ordering. We proceed by induction with respect to $n$. Denote $p(t, \alpha)=\operatorname{det}\left(t I_{n}-\sum_{i=1}^{m} \alpha_{i} A_{i}\right)$. By Lemma 2.1 $p(t, \alpha)$ splits in $F\left[\alpha_{1}, \ldots, \alpha_{m}\right][t]$ into a product of powers of distinct monic irreducible polynomials and one of the following 3 possibilities occurs.

Case 1: $p(t, \alpha)=p_{1}^{k_{1}}(t, \alpha) \cdot p_{2}^{k_{2}}(t, \alpha) \cdot p_{3}^{k_{3}}(t, \alpha) \Gamma$ where $p_{i}(t, \alpha)(i=1,2,3)$ are linear. By Lemma $2.2 \Gamma V$ includes an $\bar{\ell}$-spect subspace $\hat{V}$ of dimension $m-1$ where $\ell=1$ or 2 . By theorems 3.1 and $3.2 \Gamma \operatorname{dim} \hat{V} \leq \frac{n(n-1)}{2}+2$ and the assertion follows. 
Case 2: $p(t, \alpha)=p_{1}^{k_{1}}(t, \alpha) \cdot p_{2}^{k_{2}}(t, \alpha) \Gamma$ where $p_{1}(t, \alpha)$ is quadratic and $p_{2}(t, \alpha)$ is linear.

If $k_{1}=1 \Gamma$ then $k_{2}=n-2$ Tand by Corollary 2.9 (taking $r=n-2$ ) we have

$$
\operatorname{dim} V \leq \frac{1}{2}(n-2)(n-3)+n \cdot 2+1=\frac{n(n-1)}{2}+4 .
$$

Now the theorem follows for $n=4$. Suppose $n>4$ and $k_{1}>1$. We have a similar claim as in the proof of Theorem 3.2.

Claim: There do not exist $1 \leq r<s \leq m$ and $2 \leq \ell \leq n$ such that $d_{\Gamma}\left(A_{r}\right)=(\ell, 1)$ and $d_{\Gamma}\left(A_{s}\right)=(1, \ell)$.

Proof of claim: Denote $\hat{\alpha}=\alpha_{r} e_{r}+\alpha_{s} e_{s} \in F^{m}$.

We have $p_{1}(t, \hat{\alpha})=t^{2}+q_{1}\left(\alpha_{r}, \alpha_{s}\right) t+q_{2}\left(\alpha_{r}, \alpha_{s}\right) \Gamma p_{2}(t, \hat{\alpha})=t+\mu\left(\alpha_{r}, \alpha_{s}\right) \Gamma$ where $q_{1}\left(\alpha_{r}, \alpha_{s}\right) \Gamma q_{2}\left(\alpha_{r}, \alpha_{s}\right)$ and $\mu\left(\alpha_{r}, \alpha_{s}\right)$ are of the form

$$
\begin{aligned}
& q_{1}\left(\alpha_{r}, \alpha_{s}\right)=a_{1} \alpha_{r}+a_{2} \alpha_{s}, \\
& q_{2}\left(\alpha_{r}, \alpha_{s}\right)=a_{11} \alpha_{r}^{2}+a_{12} \alpha_{r} \alpha_{s}+a_{22} \alpha_{s}^{2}, \\
& \mu\left(\alpha_{r}, \alpha_{s}\right)=b_{1} \alpha_{r}+b_{2} \alpha_{s} .
\end{aligned}
$$

Clearly $a_{2} \Gamma a_{22}$ and $b_{2}$ must vanish.

We have $\operatorname{det}\left(\alpha_{r} A_{r}+\alpha_{s} A_{s}\right)= \pm\left(a_{11} \alpha_{r}^{2}+a_{12} \alpha_{r} \alpha_{s}\right)^{k_{1}}\left(b_{1} \alpha_{r}\right)^{k_{2}}$. Suppose $b_{1} \neq 0$. Since $\operatorname{det}\left(\alpha_{r} A_{r}+\alpha_{s} A_{s}\right)$ is either linear or independent of $\alpha_{s} \Gamma a_{12}$ must vanish. Thus $q_{1}\left(\alpha_{r}, \alpha_{s}\right) \Gamma q_{2}\left(\alpha_{r}, \alpha_{s}\right)$ and $\mu\left(\alpha_{r}, \alpha_{s}\right)$ are independent of $\alpha_{s}$ which contradicts Lemma 2.6.

Suppose $b_{1}=0$. Then $q_{1}\left(\alpha_{r}, \alpha_{s}\right)=a_{1} \alpha_{r} \Gamma q_{2}\left(\alpha_{r}, \alpha_{s}\right)=a_{11} \alpha_{r}^{2}+a_{12} \alpha_{r} \alpha_{s}$ and $\mu\left(\alpha_{r}, \alpha_{s}\right)=0$. Thus $p(t, \hat{\alpha})=\left(t^{2}+a_{1} \alpha_{r} t+a_{11} \alpha_{r}^{2}+a_{12} \alpha_{r} \alpha_{s}\right)^{k_{1}} \cdot t^{k_{2}}$. The coefficient of $t^{n-4}$ in $p(t, \hat{\alpha})$ equals $\sigma_{4}\left(\alpha_{r} A_{r}+\alpha_{s} A_{s}\right) \Gamma$ where $\sigma_{4}\left(\alpha_{r} A_{r}+\alpha_{s} A_{s}\right)$ is the sum of the principal minors of order 4 of the matrix $\alpha_{r} A_{r}+\alpha_{s} A_{s}$ Twhich must be either linear or independent of $\alpha_{s}$. Thus $a_{12}$ must vanish. Again we contradict Lemma 2.6.

As in the proof of Theorem $3.2 \Gamma$ we define $V_{1}=\operatorname{span}\left(\left\{A_{1}, \ldots, A_{m}\right\} \backslash S\right) \Gamma$ where

$$
S=\left\{A_{i}: d_{\Gamma}\left(A_{i}\right)=(1, q) \text { or } d_{\Gamma}\left(A_{i}\right)=(p, 1) \text { for some } 2 \leq p, q \leq n\right\}
$$

and $\hat{V}$ is the subspace of $M_{n-1}(F)$ obtained from $V_{1}$ by deleting the first row and column of every matrix of $V_{1}$.

i) If there exists $1 \leq j \leq m$ such that $d_{\Gamma}\left(A_{j}\right)=(1,1) \Gamma$ then there exists an $\bar{\ell}$-spect subspace $\hat{V}$ of $M_{n-1}(F)$ Twhere $\ell=1$ or 2 such that $\operatorname{dim} \hat{V} \geq \operatorname{dim} V-n$. By theorems 3.1 and $3.2 \operatorname{dim} \hat{V} \leq \frac{(n-1)(n-2)}{2}+2$ Thence $\operatorname{dim} V \leq \frac{n(n-1)}{2}+3$.

ii) If there is no $1 \leq j \leq m$ such that $d_{\Gamma}\left(A_{j}\right)=(1,1) \Gamma$ then there exists an $\bar{\ell}$-spect subspace $\hat{V}$ of $M_{n-1}(F)$ Wwhere $\ell=1,2$ or 3 such that $\operatorname{dim} \hat{V} \geq \operatorname{dim} V-(n-1)$. If $\ell=3$ then by our induction hypothesis $\operatorname{dim} \hat{V} \leq \frac{(n-1)(n-2)}{2}+4$ Thence $\operatorname{dim} V \leq \frac{n(n-1)}{2}+4$. If $\ell=1,2$ then the conclusion follows using theorems 3.1 and 3.2.

Case 3: $p(t, \alpha)=q^{k}(t, \alpha) \Gamma$ where $q(t, \alpha)$ is cubic. Here $n \equiv 0(\bmod 3)(\operatorname{son} n \geq 6)$. We have the same claim as in the previous case. In this case $\Gamma$

$$
q(t, \hat{\alpha})=t^{3}+q_{1}\left(\alpha_{r}, \alpha_{s}\right) t^{2}+q_{2}\left(\alpha_{r}, \alpha_{s}\right) t+q_{3}\left(\alpha_{r}, \alpha_{s}\right),
$$


where

$$
\begin{aligned}
& q_{1}\left(\alpha_{r}, \alpha_{s}\right)=a_{1} \alpha_{r}+a_{2} \alpha_{s} \\
& q_{2}\left(\alpha_{r}, \alpha_{s}\right)=a_{11} \alpha_{r}^{2}+a_{12} \alpha_{r} \alpha_{s}+a_{22} \alpha_{s}^{2}, \\
& q_{3}\left(\alpha_{r}, \alpha_{s}\right)=b_{1} \alpha_{r}^{3}+b_{2} \alpha_{r}^{2} \alpha_{s}+b_{3} \alpha_{r} \alpha_{s}^{2}+b_{4} \alpha_{s}^{3} .
\end{aligned}
$$

By similar reasoning explained before we conclude that $a_{2} \Gamma a_{22} \Gamma b_{2} \Gamma b_{3} \Gamma b_{4}$ must vanish.

Using $\sigma_{4}\left(\alpha_{r} A_{r}+\alpha_{s} A_{s}\right)$ we imply that $a_{12}$ vanishes $\Gamma$ which yields a contradiction to Lemma 2.6. As in the previous case the result follows immediately if there is $1 \leq$ $j \leq m$ such that $d_{\Gamma}\left(A_{j}\right)=(1,1)$. Suppose that there is no such $j$; then there exists a $\ell$-spect subspace $V$ of $M_{n-1}(F)$ Twhere $\ell=1,2$ or 3 such that $\operatorname{dim} \hat{V} \geq \operatorname{dim} V-(n-1)$. Suppose $\ell=3$. Since $(n-1) \not \equiv 0(\bmod 3) \Gamma$ then $V$ belongs to either case 1 or case 2 . Thus $\operatorname{dim} \hat{V} \leq \frac{(n-1)(n-2)}{2}+4$ and the conclusion follows. If $\ell=1$ or 2 the conclusion follows using theorems 3.1 and 3.2 .

Finally Twe give the following simple proof for the case $k=n-1$.

THEOREM 3.4. Let $F$ be a field with $\operatorname{char}(F)=0$ and $n \geq 5$. Let $V$ be $a$ $\overline{(n-1)}$-spect subspace of $M_{n}(F)$. Then $\operatorname{dim} V \leq\left(\begin{array}{c}n \\ 2\end{array}\right)+\left(\begin{array}{c}n-1 \\ 2\end{array}\right)+1$.

Proof. We can assume $I_{n} \in V$. Let $\left\{A_{1}, \ldots, A_{m}\right\}$ be some basis of $V$ in which $A_{m}=I_{n}$. Denote $p(t, \alpha)=\operatorname{det}\left(t I_{n}-\sum_{i=1}^{m} \alpha_{i} A_{i}\right)$.

By Lemma 2.1 $\Gamma(t, \alpha)$ splits into the following product

$$
p(t, \alpha)=q_{1}^{2}(t, \alpha) \cdot q_{2}(t, \alpha) \cdots q_{r}(t, \alpha),
$$

where $q_{1}, \ldots, q_{r}$ are distinct irreducible polynomials in $F\left[\alpha_{1}, \ldots, \alpha_{m}\right][t]$ and $q_{1}$ is monic and linear. Now the assertion follows from Corollary 2.9 taking $r=2$.

REMARK 3.5. Let $V$ be a subspace of $M_{n}(F)$ consisting of all matrices of the form

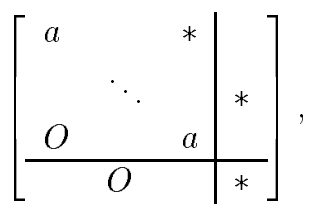

where $a$ and stars are arbitrary elements of $F$ Tand the block in the lower right corner has order $k-1$. Clearly $V$ is a $\bar{k}$-spect subspace of dimension $\left(\begin{array}{l}n \\ 2\end{array}\right)+\left(\begin{array}{l}k \\ 2\end{array}\right)+1$.

This shows that the upper bounds given in theorems 3.2 T 3.3 and 3.4 are sharp (for the appropriate $k$ ).

\section{REFERENCES}

[A] M. D. Atkinson, Spaces of matrices with several zero eigenvalues, Bull. London Math. Soc., 12:89-95 (1980).

[BC] R. Brualdi and K. Chavey, Linear spaces of Toeplitz and nilpotent matrices, J. Combin. Theory, Ser. A, 63:65-78 (1993).

[G] M. Gerstenhaber, On nilalgebras and linear varieties of nilpotent matrices, I, Amer. J. Math., $80: 614-622(1958)$. 
[OS] M. Omladič and P. Šemrl, Matrix spaces with a bounded number of eigenvalues, Linear Algebra Appl., 249:29-46 (1996).

[Se] V. N. Serezkin, Linear transformations preserving nilpotency (in Russian), Vestnik Beloruss. Gos. Univ. Ser. I Fiz. Mat. Mekh., 125:46-50 (1985). 